\title{
AMPK and SREBP-1c mediate the anti-adipogenic effect of $\beta$-hydroxyisovalerylshikonin
}

\author{
JONG-HYEOK HA, JAEWOONG JANG, SANG-IN CHUNG and YOOSIK YOON \\ Department of Microbiology, Chung-Ang University College of Medicine, Seoul 156-756, Republic of Korea
}

Received February 17, 2015; Accepted January 26, 2016

DOI: 10.3892/ijmm.2016.2484

\begin{abstract}
HIVS), which is a natural naphthoquinone compound, is one of the main chemicals isolated from a therapeutic plant, Lithospermum erythrorhizon. In the present study, we demonstrated that $\beta$-HIVS inhibited the adipogenesis of 3T3-L1 cells through AMP-activated protein kinase (AMPK)-mediated modulation of sterol regulatory element binding protein (SREBP)-1c. The anti-adipogenic effect of $\beta$-HIVS was accompanied by the increased phosphorylation of AMPK and precursor SREBP-1c. In $\beta$-HIVS-treated 3T3-L1 cells, AMPK was activated and phosphorylated precursor SREBP-1c, preventing the cleavage of precursor SREBP-1c to mature SREBP-1c. Expression of the fat-forming enzymes, acetyl-CoA carboxylase (ACC)1, fatty acid synthase (FAS) and stearoyl-CoA desaturase (SCD)1, which are transcribed by mature SREBP-1c, were downregulated, resulting in reduced intracellular fat accumulation. The anti-adipogenic effect of $\beta$-HIVS was significantly attenuated by AMPK knockdown. Knockdown of AMPK using siRNA decreased the phosphorylation of precursor SREBP-1c and increased the levels of mature SREBP. The levels of the fat-forming enzymes, ACC1, FAS and SCD1, as well as intracellular fat accumulation were also significantly increased by AMPK knockdown. These results suggest that $\beta$-HIVS activated AMPK, which was followed by the downregulation of mature SREBP-1c and fat-forming enzymes, leading to the inhibition of adipogenesis.
\end{abstract}

Correspondence to: Professor Yoosik Yoon, Department of Microbiology, Chung-Ang University College of Medicine, 84 Heukseok-ro, Dongjak-gu, Seoul 156-756, Republic of Korea

E-mail: thanks@cau.ac.kr

Abbreviations: ACC, acetyl-CoA carboxylase; AMPK, AMP-activated protein kinase; $\mathrm{C} / \mathrm{EBP} \alpha, \mathrm{CCAAT} /$ enhancer-binding protein $\alpha$; FABP, fatty acid-binding protein; FAS, fatty acid synthase; $\beta$-HIVS, $\beta$-hydroxyisovalerylshikonin; PPAR $\gamma$, peroxisome proliferator-activated receptor $\gamma$; SCD, stearoyl-CoA desaturase; SREBP, sterol regulatory element binding protein

Key words: AMP-activated protein kinase, sterol regulatory element binding protein-1c, 3T3-L1, $\beta$-hydroxyisovalerylshikonin, adipogenesis

\section{Introduction}

Obesity is defined by the excessive formation of adipose tissue, and thus an understanding of the molecular mechanism behind adipose tissue formation, i.e., adipogenesis, is necessary to find ways of preventing and treating obesity and obesity-related diseases including type 2 diabetes, hyperlipidemia and cardiovascular disease (1). Adipogenesis is mediated by the coordinated expression of the genes involved in adipocyte differentiation and intracellular fat accumulation (2-4). The 3T3-L1 cell line has been widely used as an in vitro model of adipogenesis, and the treating these cells with 3-isobutyl1-methylxanthine, dexamethasone and insulin is known to upregulate the major transcription factors of adipogenesis, peroxisome proliferator-activated receptor $\gamma(\operatorname{PPAR} \gamma)$ and CCAAT/enhancer-binding protein $\alpha(\mathrm{C} / \mathrm{EBP} \alpha)$, which directly transcribe various adipocyte marker genes, including fatty acid-binding protein (FABP) 4 (2-4).

AMP-activated protein kinase (AMPK) is a major regulator of cellular energy homeostasis (5), and it regulates carbohydrate and fat metabolism in order to maintain the cellular energy balance (6-8). AMPK is activated by the increased ratio of AMP/ATP, and it is known to phosphorylate various target proteins involved in cell growth and metabolism (9). Dysregulation of AMPK is involved in various disorders including metabolic diseases, cardiovascular disease, cancer and dementia (10). Recently, Li et al reported that sterol regulatory element binding protein (SREBP)1c was one of the target proteins directly phosphorylated by AMPK (11). AMPK was found to phosphorylate the Ser372 residue of SREBP-1c, which inhibited the proteolytic cleavage of the precursor form of SREBP-1c (precursor SREBP-1c) into mature SREBP-1c, resulting in the inhibition of hepatic steatosis in insulin-resistant mice. SREBP-1c has also been identified as one of the transcription factors involved in adipogenesis (12). However, to the best of our knowledge, AMPK-mediated modulation of SREBP-1c has never been suggested to be the anti-adipogenic mechanism of any compound which inhibits adipogenesis.

Shikonin is a major chemical ingredients of Lithospermum erythrorhizon, a therapeutic plant used for the treatment of macular eruption, measles, sore-throat, carbuncles and burns (13). Lithospermum erythrorhizon contains various shikonin compounds, including shikonin, acetylshikonin, isobutyrylshikonin and $\beta$-hydroxyisovalerylshikonin ( $\beta$-HIVS) (14). In the present study, we demonstrated the anti-adipogenic effect 
of $\beta$-HIVS and elucidated its molecular mechanisms. It was found that $\beta$-HIVS activated AMPK, resulting in the increased phosphorylation of SREBP-1c, preventing its proteolytic maturation. This was followed by the reduced expression of fat-forming enzymes, including acetyl-CoA carboxylase (ACC)1, fatty acid synthase (FAS) and stearoyl-CoA desaturase (SCD)1, which resulted in reduced intracellular fat accumulation.

\section{Materials and methods}

Chemicals and reagents. The cell culture reagents, Dulbecco's modified Eagle's medium (DMEM), fetal bovine serum (FBS) and penicillin/streptomycin, were all obtained from Life Technologies (Grand Island, NY, USA). Anti-PPAR $\gamma$ (\#2430), anti-FABP4 (\#2120), anti-p-AMPK (\#2535), anti-AMPK (\#2603), anti-FABP4 (\#2120) and anti-p-SREBP1c (\#9847) antibodies, and anti-mouse (\#7076S)/anti-rabbit (\#7074) secondary antibody were all purchased from Cell Signaling Technology (Beverly, MA, USA). Anti-C/EBP $\alpha$ (sc-61) and anti- $\beta$-actin (sc-47778) antibodies were all purchased from Santa Cruz Biotechnology, Inc (Santa Cruz, CA, USA). Anti-SREBP1c (557036) was purchased from BD science (Franklin Lakes, NJ, USA). AMPK small interfering (si)RNA and control siRNA were both purchased from Santa Cruz Biotechnology, Inc. Lipofectamine RNAiMAX transfection reagent was purchased from Invitrogen (Carlsbad, CA, USA). Shikonin, acetylshikonin, isobutyrylshikonin and $\beta$-HIVS were all purchased from Wako Pure Chemical Industries, Ltd. (Osaka, Japan). All other chemicals were from Sigma-Aldrich (St. Louis, MO, USA).

Cell culture. The 3T3-L1 preadipocytes were purchased from the American Type Culture Collection (ATCC; Manassas, VA, USA) and subcultured every 2 days. The cells were seeded in 6 -well plates at a density of $1.5 \times 10^{5}$ cells/well. Two days after reaching confluence (day 0 ), the 3 T3-L1 cells were differentiated in a differentiation-induction medium containing DMEM, supplemented with $1 \mu \mathrm{g} / \mathrm{ml}$ insulin, $0.25 \mu \mathrm{M}$ dexamethasone, 0.5 mM 3-isobutyl-1-methylxanthine and 10\% FBS, for 2 days. The cells were then maintained in a differentiation-maintenance medium containing DMEM, supplemented with $1 \mu \mathrm{g} / \mathrm{ml}$ insulin and $10 \%$ FBS. The differentiation-maintenance medium was replaced every 2 days until the cells were harvested.

Oil Red $O$ staining of intracellular fat. The 3T3-L1 cells were seeded in 12-well plates at a density of $7.5 \times 10^{4}$ cells/well. After differentiation for 7 days, the cells were washed with phosphate-buffered saline (PBS), and incubated for $4 \mathrm{~h}$ in 4\% paraformaldehyde (Sigma-Aldrich). The cells were then washed with distilled water and stained with $0.3 \%$ Oil Red O (Sigma-Aldrich) solution for $1 \mathrm{~h}$. After washing them 3 times with distilled water, the cells were completely dried and observed under an inverted microscope (Olympus, Tokyo, Japan). Photographic images were captured and then $100 \%$ isopropanol (Sigma-Aldrich) was added to each well to extract the stained Oil Red $\mathrm{O}$ dye. The contents of the extracted dye were measured at $540 \mathrm{~nm}$ using a spectrophotometer (BioTek Instruments, Inc., Winooski, VT, USA).

Cell viability assay. Cell viability was determined using a CellCountEZ ${ }^{\mathrm{TM}}$ Cell Survival assay kit (purchased from
Rockland Immunochemicals, Limerick, PA, USA), as previously described (15); viability was determined based on the ability of viable mammalian cells to convert hydroxyethyl disulfide into mercaptoethanol. The amount of mercaptoethanol produced from hydroxyethyl disulfide can be measured in the extracellular culture media since mercaptoethanol produced inside cells is extruded quickly by cells through an active transport mechanism.

Reverse transcription-quantitative polymerase chain reaction (RT-qPCR). The 3T3-L1 cells were seeded into 12-well plates at a density of $7.5 \times 10^{4}$ cells/well, and, after differentiation, total RNA was extracted using an RNeasy kit (Qiagen, Hilden, Germany). One microgram of total RNA was reverse transcribed at $37^{\circ} \mathrm{C}$ using the cDNA reverse transcription kit (Applied Biosystems, Inc., Foster City, CA, USA). qPCR was performed using the 7000 Real-Time PCR System (Applied Biosystems) in a final volume of $20 \mu \mathrm{l}$, which included the TaqMan Gene Expression Master Mix, $250 \mathrm{nM}$ of TaqMan probe, an optimized concentration of each primer and $1 \mu \mathrm{l}$ of the reverse transcription product containing cDNA. The reaction mixtures were preheated at $95^{\circ} \mathrm{C}$ for $10 \mathrm{~min}$ to activate the enzyme and then subjected to 40 cycles of melting at $95^{\circ} \mathrm{C}$ for $15 \mathrm{sec}$ and annealing/extension at $60^{\circ} \mathrm{C}$ for $1 \mathrm{~min}$. The efficiency of RT-qPCR was approximately $100 \%$. The Assays-on-Demand gene expression products (Applied Biosystems) were used to evaluate the mRNA levels of PPAR $\gamma$ (Mm00440945_m1), C/EBP $\alpha$ (Mm01265914_s1), SREBP-1c (Mm00550338_m1), FABP4 (Mm00445880_m1), FAS (Mm01253292_m1), ACC1 (Mm01304257_m1), SCD1 (Mm00772290_m1) as well as the level of 18S rRNA (Hs99999901_s1). 18S rRNA was used as an internal control, as previously described (16). For each sample, the mRNA level was normalized against the level of $18 \mathrm{~S}$ rRNA, and the ratio of normalized mRNA in each sample to that in the control sample was determined using the comparative $\mathrm{Ct}$ method (17).

Protein extraction and western blot analysis. Cells were harvested using a cell scraper in ice-cold PBS, and lysed with RIPA buffer containing $25 \mathrm{mM}$ Tris- $\mathrm{HCl}$ (pH 7.6), $150 \mathrm{mM}$ $\mathrm{NaCl}, 1 \%$ Nonidet $\mathrm{P}-40,1 \%$ sodium deoxycholate, $0.1 \%$ SDS and a protease inhibitor cocktail (Sigma-Aldrich) for $30 \mathrm{~min}$ at $4^{\circ} \mathrm{C}$. The total cell lysates were then obtained after centrifuging at $20,000 \times \mathrm{g}$ for $20 \mathrm{~min}$ at $4^{\circ} \mathrm{C}$ to remove the insoluble materials. The protein concentrations were determined using a BCA protein assay kit (Pierce, Rockford, IL, USA). Twenty micrograms of protein were separated using $10 \%$ polyacrylamide gel electrophoresis and electrotransferred onto nitrocellulose membranes at $180 \mathrm{~mA}$ for $1 \mathrm{~h}$. The membranes were then blocked for $2 \mathrm{~h}$ at room temperature with Tris-buffered saline (TBS) containing 5\% skim milk. Blocked nitrocellulose membranes were incubated with TBS containing $0.1 \%$ Tween-20 and a 1:1,000-dilution of primary antibody overnight at $4{ }^{\circ} \mathrm{C}$ followed by a 1:1,000-dilution of horseradish peroxidase-conjugated secondary antibody for $1 \mathrm{~h}$ at room temperature. Peroxidase activity was visualized using an ECL kit (Pierce).

Transfection with siRNA. Two days after reaching confluence, 3T3-L1 cells were incubated in serum-free medium for $1 \mathrm{~h}$ and 

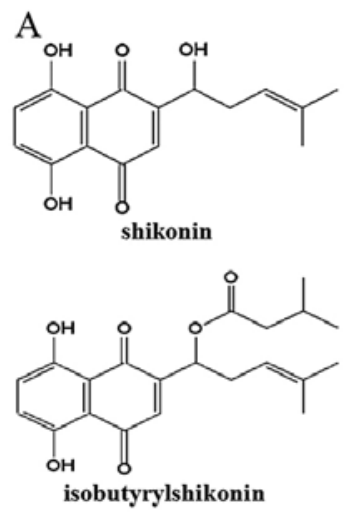
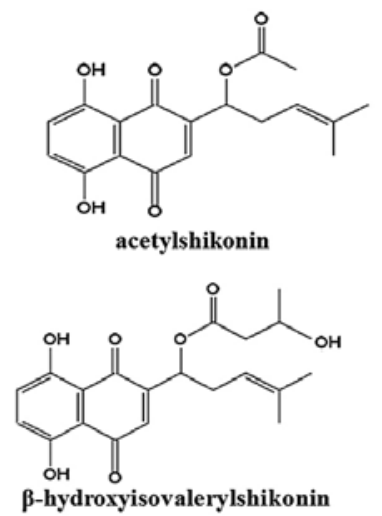

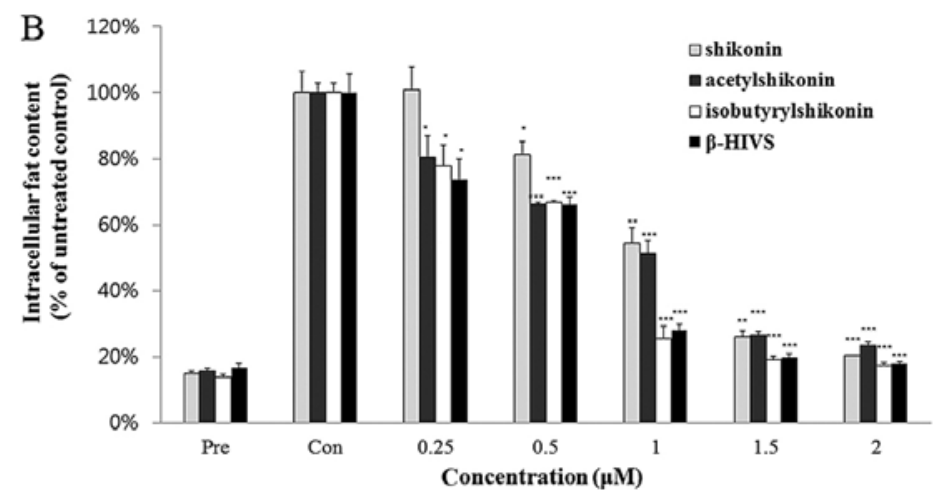

Figure 1. Comparison of the anti-adipogenic activities of shikonin compounds. (A) Chemical structures of shikonin compounds contained in Lithospermum erythrorhizon. (B) The inhibitory effect of shikonin compounds on intracellular fat accumulation. Intracellular fat droplets were stained with Oil Red $\mathrm{O}$ and extracted for measurement by spectrophotometric analysis at $540 \mathrm{~nm}$ on day 7 of $3 \mathrm{~T} 3-\mathrm{L} 1$ adipocyte differentiation. ${ }^{*} \mathrm{P}<0.05,{ }^{* *} \mathrm{P}<0.01,{ }^{* * *} \mathrm{P}<0.001$ compared with untreated adipocytes (Con). Pre denotes undifferentiated preadipocytes.

then transfected with $60 \mathrm{nM}$ of AMPK siRNA or $60 \mathrm{nM}$ of control siRNA using Lipofectamine RNAiMAX transfection reagent. Six hours later, the transfected cells were differentiated by replacing the medium with differentiation-induction medium. Total RNA and protein were extracted for RT-qPCR and western blot analysis, respectively.

Analysis of nuclear SREBP-1c levels. In the present study, the cells were harvested using cell scrapers, and the nuclear extracts were prepared using a nuclear extract kit (Active Motif, Carlsbad, CA, USA). Protein concentrations in the nuclear extracts were determined using a BCA protein assay kit (Pierce). Twenty micrograms of nuclear protein were separated using $10 \%$ polyacrylamide gel electrophoresis and analyzed by western blot analysis using an anti-SREBP-1c antibody followed by secondary antibody.

Statistical analyses. All data are represented as the means \pm standard deviation (SD) of at least three replicated experiments. Statistically significant differences between treated and untreated samples were detected using unpaired t-tests. All analyses were performed using SPSS v.14 (SPSS, Inc., Chicago, IL, USA). A P-value $<0.05$ was considered to indicate a statistically significant difference.

\section{Results}

Comparison of the anti-adipogenic effect of various shikonin compounds. It has previously been reported that Lithospermum erythrorhizon contains various shikonin compounds, including shikonin, acetylshikonin, isobutyrylshikonin and $\beta$-hydroxy-isovalerylshikonin ( $\beta$-HIVS) (14). These shikonin compounds have the common basic structure of naphthoquinone, while the attached chemical groups are slightly different (Fig. 1A). To determine which compound exerted the greatest anti-adipogenic effect, the 3T3-L1 cells were treated with the four shikonin compounds at various concentrations for 7 days. Analysis of the effects on intracellular fat content revealed that isobutyrylshikonin and $\beta$-HIVS exerted a greater anti-adipogenic effect than shikonin and acetylshikonin (Fig. 1B). However, the intracellular fat content of 3T3-L1 cells treated with isobutyrylshikonin or $\beta$-HIVS for 7 days were similar. Thus, in the subsequent experiment, 3T3-L1 cells were treated with isobutyrylshikonin or $\beta$-HIVS on days 0-2 , 3-4 and 4-7 of adipogenic differentiation (Fig. 2). The anti-adipogenic effect of $\beta$-HIVS was found to be slightly greater than that of isobutyrylshikonin (Fig. 2C and D). These data indicate that $\beta$-HIVS exerted the greatest anti-adipogenic effect among the shikonin compounds, and further studies were therefore conducted using $\beta$-HIVS.

Anti-adipogenic effect of $\beta$-HIVS. In order to determine the most effective concentration of $\beta$-HIVS required for anti-adipogenic activity, the 3T3-L1 cells were treated with $\beta$-HIVS at various concentrations $(0.25,0.5,1,1.5$ and $2 \mu \mathrm{M})$ for 7 days. The Oil Red O staining revealed that intracellular fat droplet formation was inhibited by $\beta$-HIVS in a dose-dependent manner, and that $\beta$-HIVS markedly inhibited fat droplet formation at a concentration of $2 \mu \mathrm{M}$, almost to the control level (Fig. 3A and B). Cytotoxicity was not observed when the 3T3-L1 cells were treated with $\beta$-HIVS at concentrations of up to $2 \mu \mathrm{M}$ for 7 days, and cell viability was maintained at $>90 \%$ of the untreated control cells (Fig. 3C), indicating that the inhibitory effects of $\beta$-HIVS on fat accumulation were not mediated by cytotoxicity. Further experiments were conducted with $\beta$-HIVS at a concentration of $2 \mu \mathrm{M}$.

During adipogenesis of the 3T3-L1 cells, the mRNA expression of the fat-forming enzymes, ACC1, FAS and SCD1, was upregulated, and this effect was significantly reduced by $\beta$-HIVS treatment (Fig. 4A-C). The major transcription factors of adipogenesis, PPAR $\gamma$ and $\mathrm{C} / \mathrm{EBP} \alpha$, which are markedly upregulated during adipogenesis and are involved in the transcription of adipocyte marker genes including FABP4, were also significantly reduced by $\beta$-HIVS treatment (Fig. 4D-F).

It is noteworthy that ACC1, FAS and SCD1 have been reported to be transcribed by a transcription factor, SREBP-1c. It has also been reported that the mRNA expression of SCD1 is induced by the binding of nuclear SREBP-1c to its promoter (18), and SREBP-1c is the transcription factor that binds to a regulatory element in the enhancer of the FAS gene (19). The level of ACC1 mRNA is also controlled by the binding of SREBP-1c to the ACC1 promoter $(19,20)$. It has 

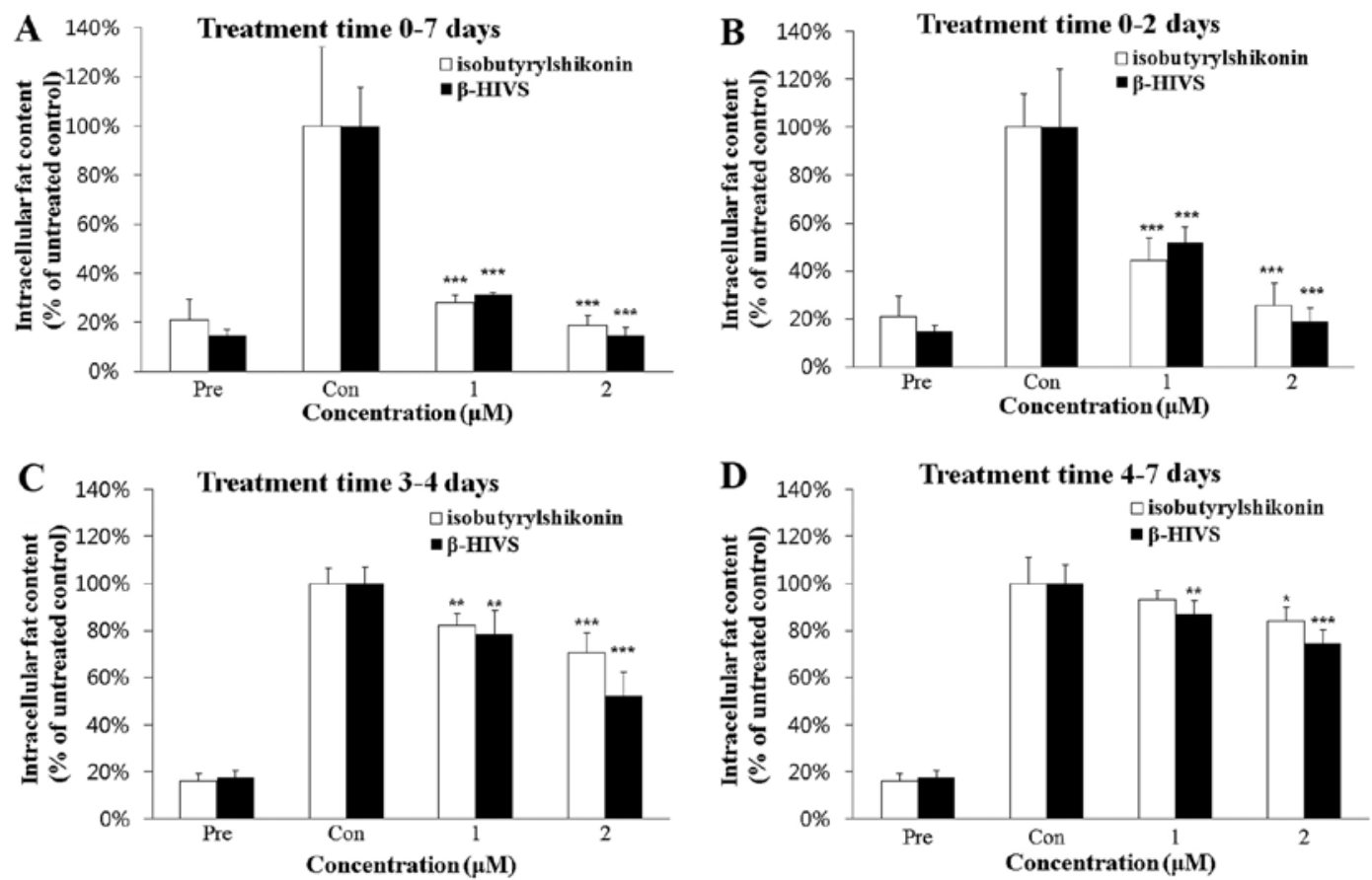

Figure 2. Comparison of the anti-adipogenic activity of isobutyrylshikonin and $\beta$-hydroxyisovalerylshikonin ( $\beta$-HIVS). The 3T3-L1 cells were treated with $\beta$-HIVS or isobutyrylshikonin during days (A) 0-7, (B) 0-2, (C) 3-4 and (D) 4-7. Intracellular fat droplets were stained with Oil Red O and extracted for measurement by spectrophotometric analysis at $540 \mathrm{~nm}$ on day 7 of $3 \mathrm{~T} 3-\mathrm{L} 1$ adipocyte differentiation. ${ }^{*} \mathrm{P}<0.05,{ }^{* *} \mathrm{P}<0.01,{ }^{* * * *} \mathrm{P}<0.001$ compared with the untreated adipocytes (Con). Pre denotes undifferentiated preadipocytes.
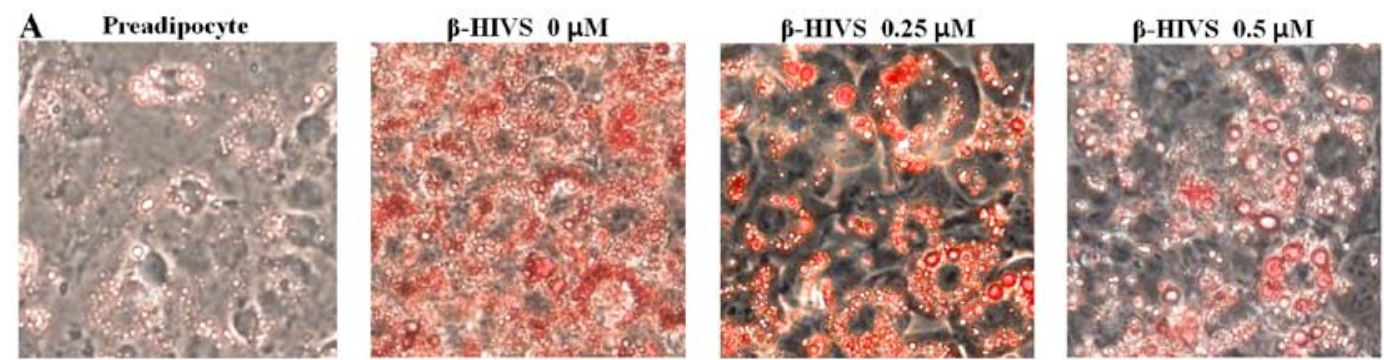

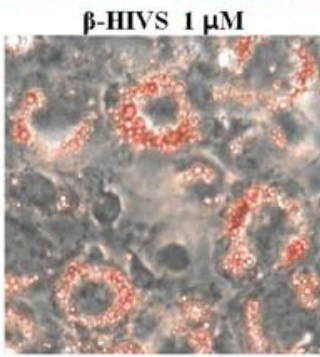

B

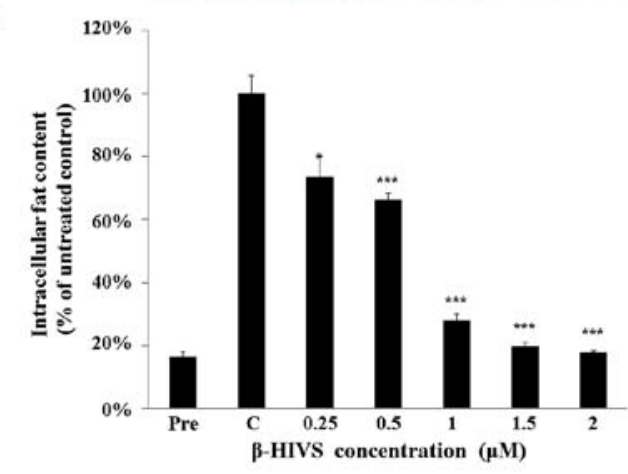

B-HIVS $1.5 \mu \mathrm{M}$ uM

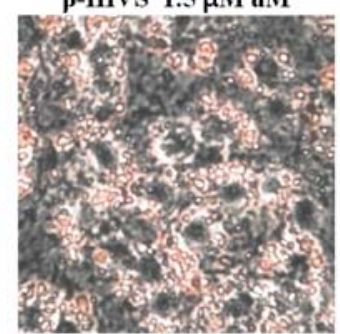

C $120 \%$
B-HIVS $2 \mu \mathrm{M}$

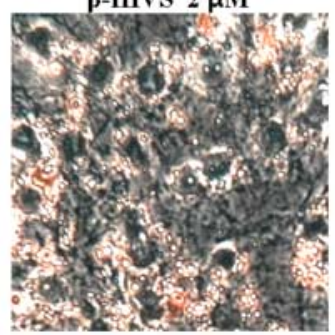

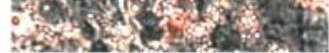

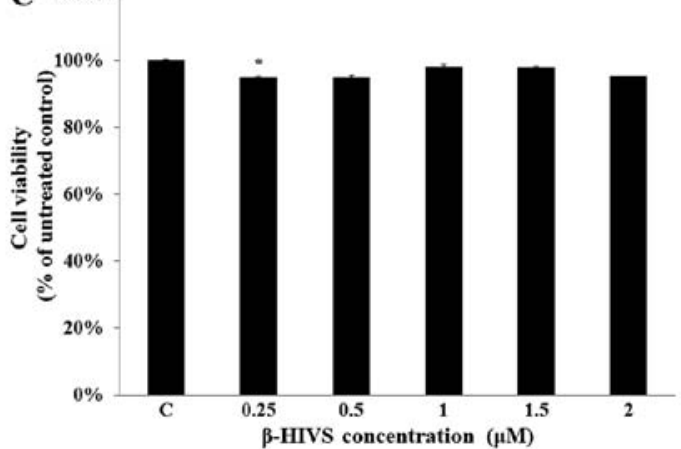

Figure 3. Anti-adipogenic activity and cytotoxicity of $\beta$-hydroxyisovalerylshikonin ( $\beta$-HIVS). (A) Intracellular fat droplets were stained with Oil Red O on day 7 of 3T3-L1 adipocyte differentiation and examined using light microscopy. (B) Stained Oil Red O dye was extracted by isopropyl alcohol and measured by a spectrophotometer at an optical density of $540 \mathrm{~nm}$. (C) Cell viability was measured on day 7 of $3 \mathrm{~T} 3-\mathrm{L} 1$ adipocyte differentiation. ${ }^{*} \mathrm{P}<0.05,{ }^{* * *} \mathrm{P}<0.001$ compared with the untreated adipocytes. Pre denotes undifferentiated preadipocytes. 

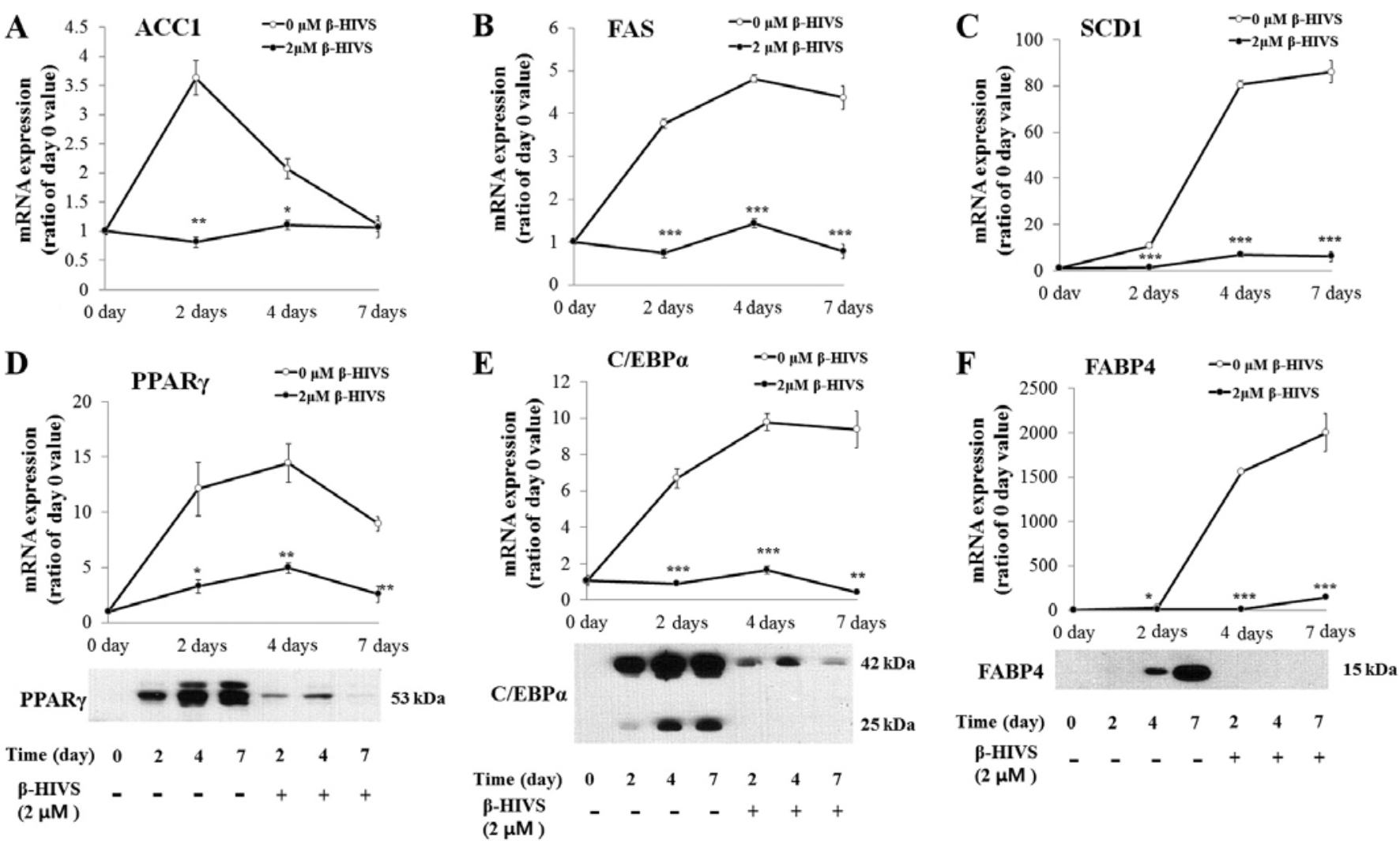

Figure 4. Effects of $\beta$-hydroxyisovalerylshikonin ( $\beta$-HIVS) on the expression of fat-forming enzymes and adipocyte-specific genes. (A-C) Effects of $2 \mu \mathrm{M}$ of $\beta$-HIVS on the mRNA expression of fat-forming enzymes, acetyl-CoA carboxylase (ACC)1, fatty acid synthase (FAS) and stearoyl-CoA desaturase (SCD)1, in 3T3-L1 cells differentiated for 0, 2, 4 and 7 days. (D-F) Effects of $2 \mu \mathrm{M}$ of $\beta$-HIVS on the mRNA and protein expression of adipocyte-specific genes such as major adipogenic transcription factors, peroxisome proliferator-activated receptor $\gamma(\mathrm{PPAR} \gamma)$ and CCAAT/enhancer-binding protein $\alpha(\mathrm{C} / \mathrm{EBP} \alpha)$, and an adipocyte marker gene, fatty acid-binding protein (FABP) 4, in 3T3-L1 cells differentiated for $0,2,4$ and 7 days. ${ }^{*} \mathrm{P}<0.05,{ }^{* * *} \mathrm{P}<0.01,{ }^{* * * *} \mathrm{P}<0.001$ compared with the untreated adipocytes on the same differentiation day.

also been reported that SREBP-1c induces the expression of PPAR $\gamma$, which collaborates with $\mathrm{C} / \mathrm{EBP} \alpha$ to transcribe adipocyte marker genes such as FABP4 (21).

Effects of $\beta$-HIVS on AMPK activation and SREBP-1c downregulation. AMPK is known to suppress adipogenesis when it is activated by phosphorylation (22). To elucidate the association between $\beta$-HIVS and the activatation of AMPK, we determined whether $\beta$-HIVS induces AMPK phosphorylation during adipogenesis, and it was found that the p-AMPK level was markedly upregulated in the $\beta$-HIVS-treated cells compared with the untreated cells. The total AMPK protein level was almost unchanged, demonstrating that $\beta$-HIVS induced the phosphorylation of AMPK without affecting its total protein level (Fig. 5A). 5-Aminoimidazole-4-carboxamide-1- $\beta$-dribofuranoside (AICAR) is the most well-known activator of AMPK, which activates AMPK by increasing its phosphorylation, and it was thus necessary to compare the AMPK-activating effect of $\beta$-HIVS with AICAR (23). The levels of p-AMPK and p-precursor-SREBP-1c, which is one of the products made by p-AMPK, were measured in order to compare the AMPK-activating effect of AICAR and $\beta$-HIVS at various concentrations (AICAR: 25, 150, 250 and $500 \mathrm{mM}$ vs. $\beta$-HIVS: $0.1,0.5,1$ and $2 \mu \mathrm{M}$ ). The results revealed that the levels of p-AMPK and p-precursor-SREBP-1c were increased by AICAR and $\beta$-HIVS (Fig. 5B). However, $\beta$-HIVS increased the levels of p-AMPK and p-precursor-SREBP-1c at a much lower concentration, $<1 / 100,000$, compared with AICAR,demonstrating that $\beta$-HIVS is a much more effective AMPK activator compared with AICAR.

Li et al previously reported that phosphorylated/activated AMPK directly phosphorylates precursor SREBP-1c at the Ser372 residue, which prevents the proteolytic maturation of precuser SREBP-1c into mature SREBP-1c and the translocation of mature SREBP-1c into the nucleus (11). When the 3T3-L1 cells were treated with $\beta$-HIVS for $6,12,24$ and $48 \mathrm{~h}$, the level of p-precursor-SREBP-1c was increased similarly to the p-AMPK level. As a result, the level of precursor SREBP-1c (unphosphorylated) and the proteolytic cleavage of precurser SREBP-1c (unphosphorylated) to mature-SREBP-1c was decreased by $\beta$-HIVS treatment. Furthermore, the level of nuclear mature SREBP-1c was also decreased, as the phosphorylation of Ser372 prevented nuclear translocation of SREBP-1c (Fig. 5C). When the 3T3-L1 cells were treated with $\beta$-HIVS for longer periods of time, of 2, 4 and 7 days, similar patterns of change were observed in the phosphorylation, proteolytic maturation and nuclear translocation of SREBP-1c. The level of precursor SREBP-1c (unphosphorylated) and its proteolytic cleavage into mature SREBP-1c, followed by its nuclear translocation, were reduced by the increase of p-precursor SREBP-1c in the $\beta$-HIVS-treated cells compared with the untreated cells (Fig. 5D). 
A

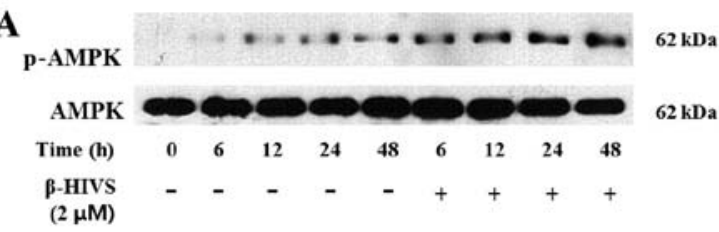

P-

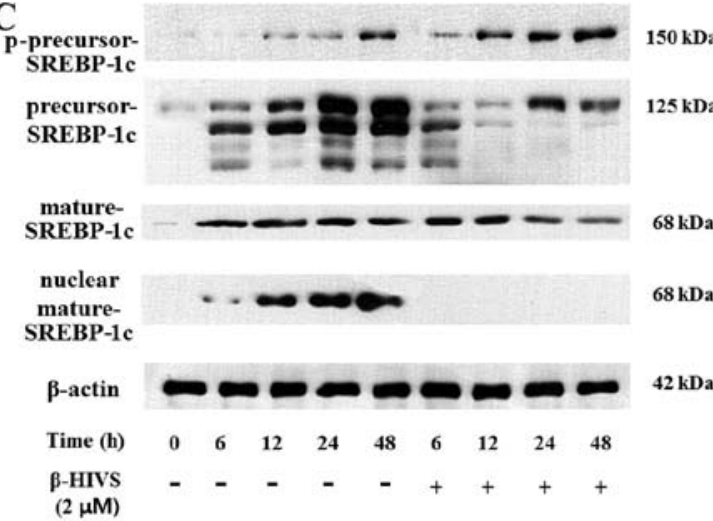

B

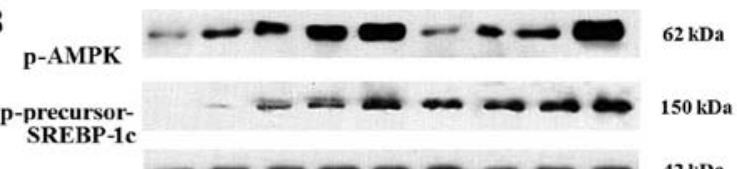

$\beta$-actin

$\operatorname{AICAR}(\mathrm{mM}) \quad-\quad 25 \quad 150 \quad 250 \quad 500 \quad-\quad-\quad-\quad-$

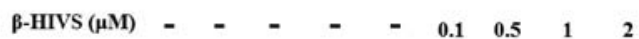

D

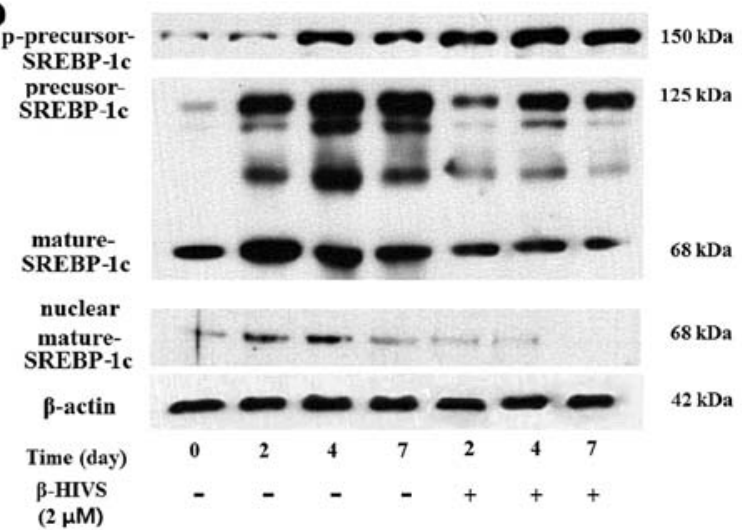

Figure 5. Effects of $\beta$-hydroxyisovalerylshikonin ( $\beta$-HIVS) on AMP-activated protein kinase (AMPK) and sterol regulatory element binding protein (SREBP)1c during adipogenesis. (A) Levels of phosphorylated (p-)AMPK and total AMPK in 3T3-L1 cells differentiated for 6, 12,24 and $48 \mathrm{~h}$ in the presence or absence of $\beta$-HIVS. (B) Levels of p-AMPK and p-precursor SREBP-1c in 3T3-L1 cells differentiated for $24 \mathrm{~h}$ in the presence of various concentrations of 5-aminoimidazole4-carboxamide-1- $\beta$-D-ribofuranoside (AICAR) and $\beta$-HIVS. (C and D) Levels of p-precursor SREBP-1c, unphosphorylated precursor/mature SREBP-1c and nuclear mature SREBP-1c in 3T3-L1 cells differentiated for 6, 12, 24 and $48 \mathrm{~h}(\mathrm{C})$ and 2, 4, 7 days (D) in the presence or absence of $\beta$-HIVS.
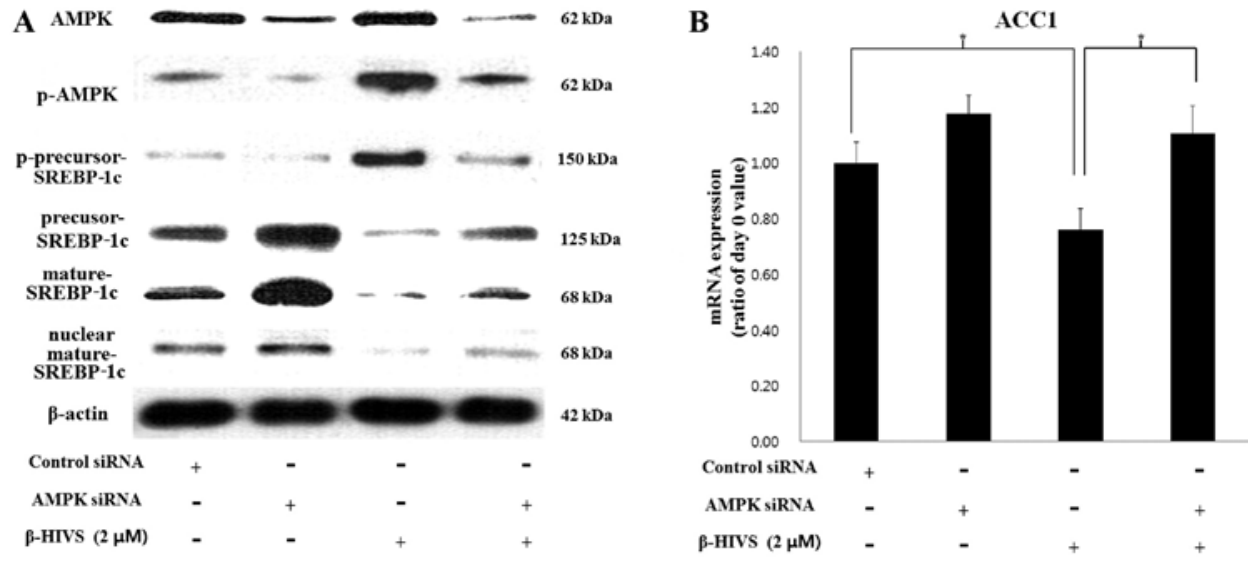

C

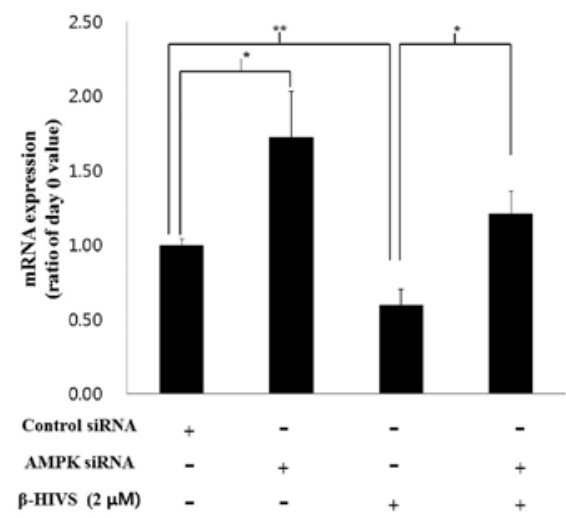

D

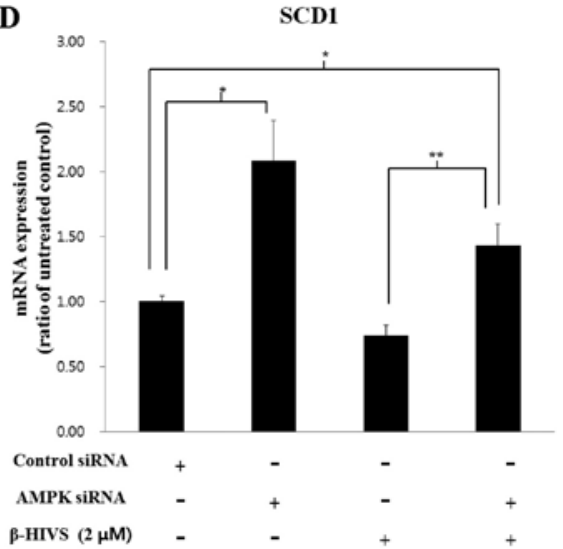

Figure 6. Effects of AMP-activated protein kinase (AMPK) knockdown on sterol regulatory element binding protein (SREBP)1c and the fat-forming enzymes (A) Effects of AMPK siRNA transfection on the protein levels of AMPK, phosphorylated (p-)AMPK, p-precursor SREBP-1c, unphosphorylated precursor/ mature SREBP-1c and nuclear mature SREBP-1c. (B-D) Effects of AMPK siRNA transfection on the mRNA levels of the fat-forming enzymes, acetyl-CoA carboxylase (ACC)1 (B), fatty acid synthase (FAS) (C) and stearoyl-CoA desaturase (SCD)1 (D). Following transfection with the control siRNA or the AMPK siRNA, the 3T3-L1 cells were differentiated for 7 days in the presence or absence of $2 \mu \mathrm{M}$ of $\beta$-hydroxyisovalerylshikonin $\left(\beta\right.$-HIVS). ${ }^{*} \mathrm{P}<0.05,{ }^{* *} \mathrm{P}<0.01$. 


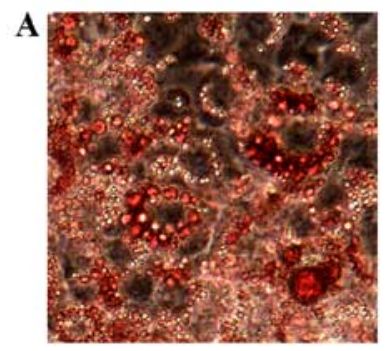

Control siRNA

$0 \mu \mathrm{M}$ B-HIVS

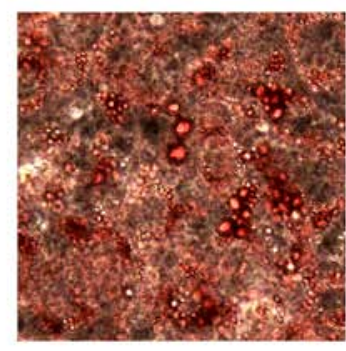

AMPK siRNA

$0 \mu \mathrm{M} \beta$-HIVS

B

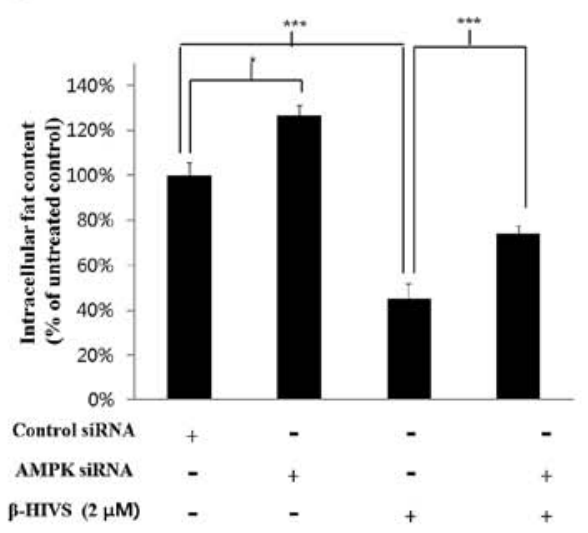

D C/EBPQ

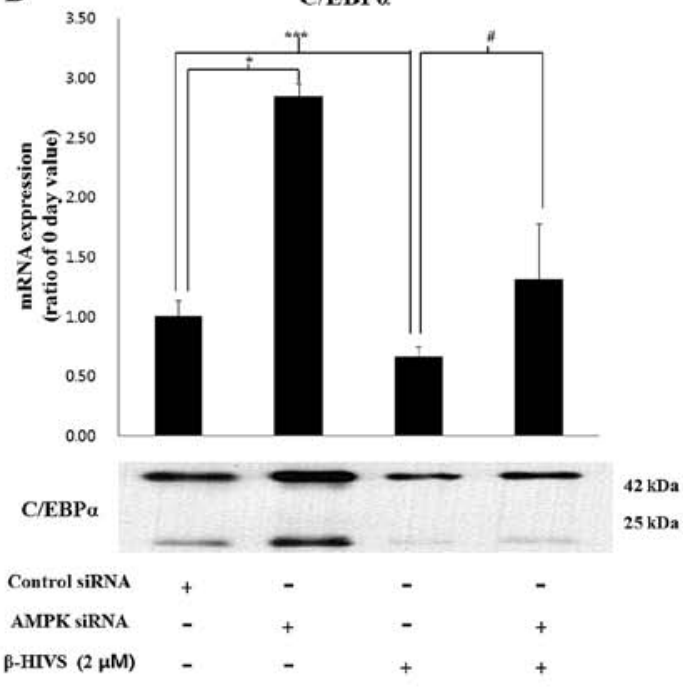

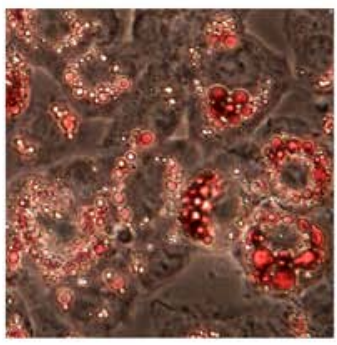
$2 \mu \mathrm{M} \beta$-HIVS
Control siRNA

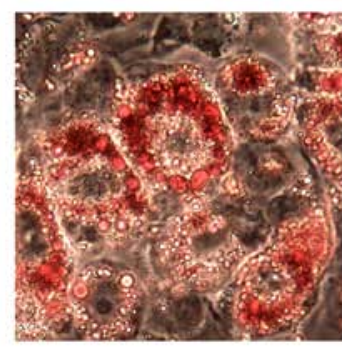

AMPK siRNA

$2 \mu \mathrm{M}$ AMPK

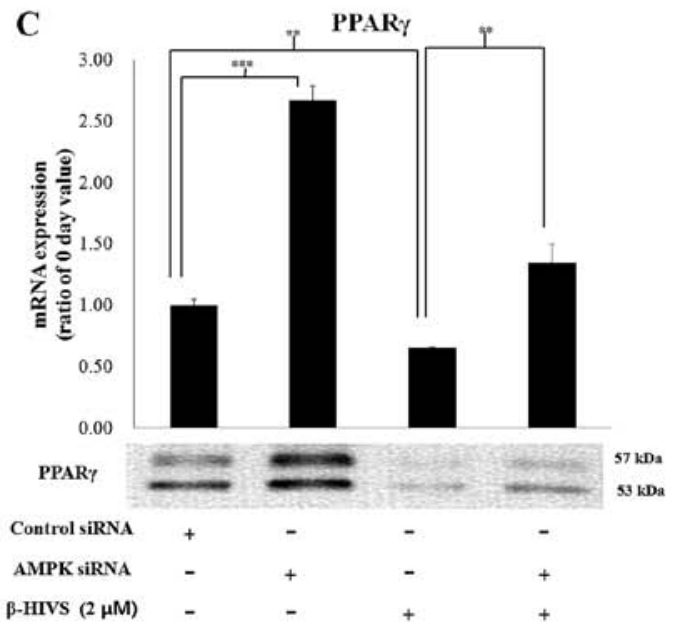

E

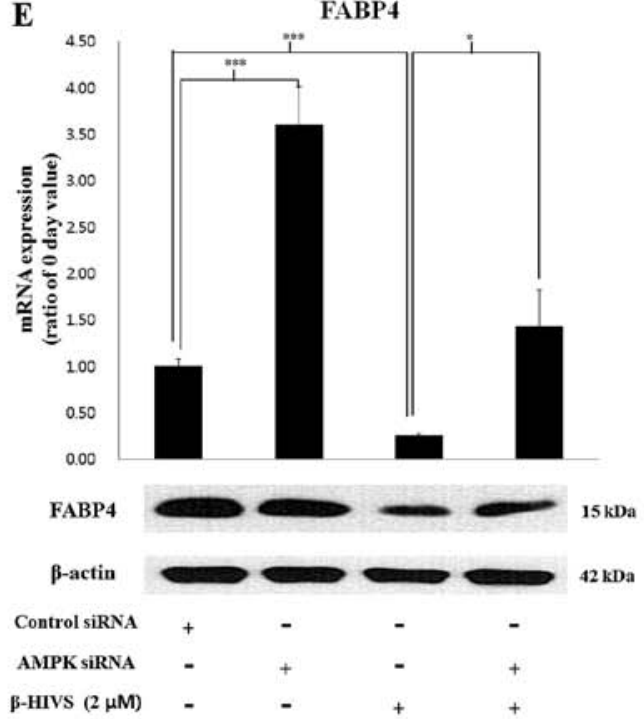

Figure 7. Effects of AMP-activated protein kinase (AMPK) knockdown on intracellular fat accumulation and adipocyte-specific gene expression. (A and B) Effects of AMPK siRNA transfection on intracellular fat accumulation. Intracellular fat droplets were stained with Oil Red O dye and examined by light microscopy (A). Oil Red O dye in the cells was extracted using isopropyl alcohol and measured by spectrophotometry at $540 \mathrm{~nm}$ (B). (C-E) Effects of AMPK siRNA transfection on the mRNA and protein levels of adipocyte-specific genes, (C) peroxisome proliferator-activated receptor $\gamma($ PPAR $\gamma$, (D) CAAT/enhancer-binding protein $\alpha(\mathrm{C} / \mathrm{EBP} \alpha)$ and (E) fatty acid-binding protein (FABP)4. Following transfection with control siRNA or AMPK siRNA, the 3T3-L1 cells were differentiated for 7 days in the presence or absence of $\beta$-hydroxyisovalerylshikonin $\left(\beta\right.$-HIVS). ${ }^{*} \mathrm{P}<0.1,{ }^{*} \mathrm{P}<0.05,{ }^{* *} \mathrm{P}<0.01,{ }^{* * *} \mathrm{P}<0.001$.

Effects of AMPK knockdown on the anti-adipogenic effect of $\beta$-HIVS. To confirm the essential role which AMPK plays in the anti-adipogenic mechanism of $\beta$-HIVS, we performed siRNA-mediated knockdown of AMPK in 3T3-L1 cells in the presence or absence of $\beta$-HIVS. The siRNA-mediated knockdown of AMPK reduced the levels of AMPK and p-AMPK. We noted that p-precursor-SREBP-1c, which is a product made by $\mathrm{p}-\mathrm{AMPK}$, was also reduced by AMPK knockdown. As a result, levels of precursor SREBP-1c (unphosphorylated), mature SREBP-1c and nuclear mature SREBP-1c were increased by AMPK knockdown (Fig. 6A). Mature SREBP-1c is a transcription factor required for the expression of the fat-forming enzymes ACC1,FAS and SCD1, the levels of which were all significantly increased by AMPK siRNA compared to transfection with control siRNA (Fig. 6B-D). SREBP-1c is also known to induce self mRNA transcription by binding to its own promoter $(24,25)$. In the present study, it was also found that the SREBP-1c mRNA level was increased by 
A

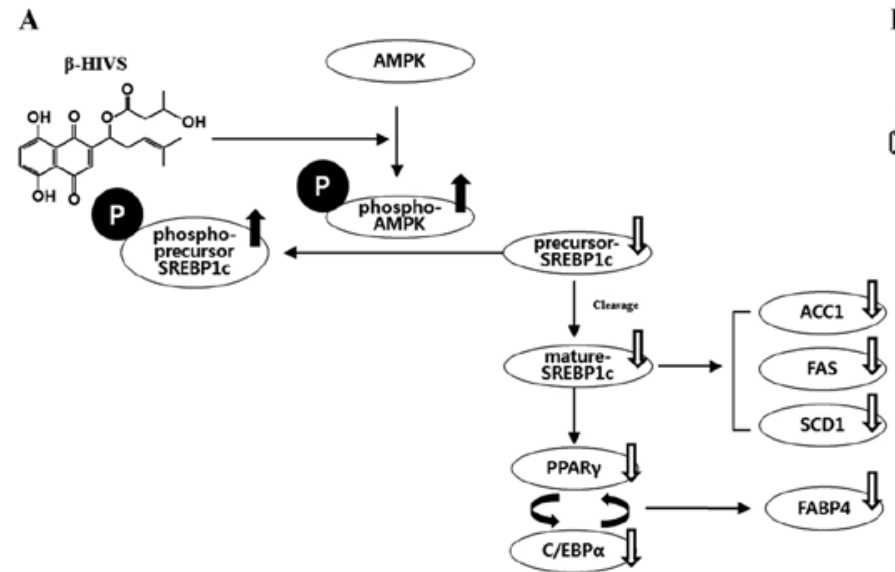

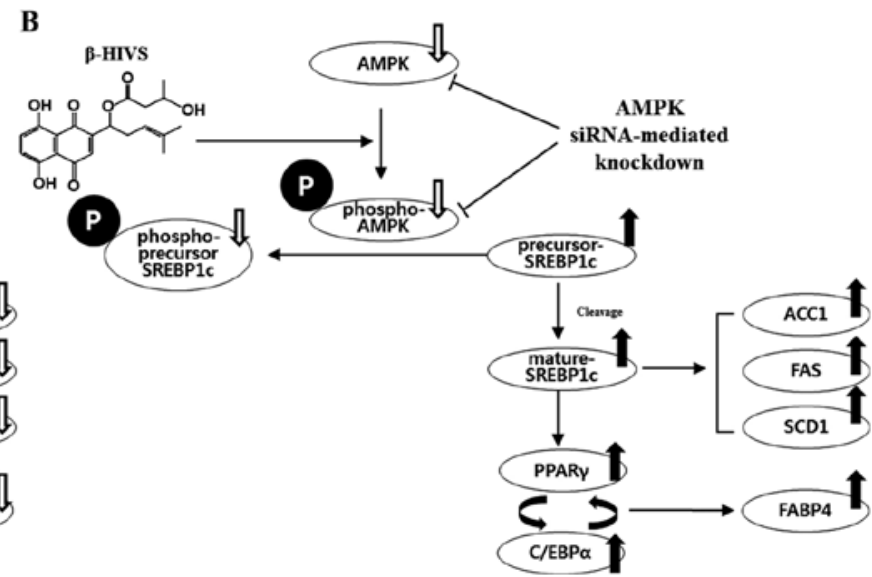

Figure 8. Molecular mechanisms for the anti-adipogenic effect of $\beta$-hydroxyisovalerylshikonin ( $\beta$-HIVS) and effect of AMP-activated protein kinase (AMPK) knockdown. (A) $\beta$-HIVS activates AMPK by increasing its phosphorylation. Phosphorylated (p-)AMPK directly phosphorylates precursor-sterol regulatory element binding protein (SREBP)-1c into p-precursor SREBP-1c, which cannot be cleaved into mature SREBP-1c, and the level of mature SREBP-1c level decreases. A decreased level of mature SREBP-1c, which is a transcription factor required for the expression of acetyl-CoA carboxylase (ACC)1, fatty acid synthase (FAS) and stearoyl-CoA desaturase (SCD)1 and peroxisome proliferator-activated receptor $\gamma$ (PPAR $\gamma$ ), leads to reduced intracellular fat accumulation. (B) AMPK siRNA reduces the levels of AMPK and p-AMPK, which leads to the reduced phosphorylation of precursor SREBP-1c and an increased level of unphosphorylated precursor SREBP-1c. Unphosphorylated precursor SREBP-1c is cleaved into mature SREBP-1c, which is a transcription factor required for the expression of ACC1, FAS, SCD1 and PPAR $\gamma$, leads to increased intracellular fat accumulation.

AMPK knockdown in $\beta$-HIVS-treated cells, possibly through the reduction in SREBP-1c phosphorylation and increase in mature SREBP-1c level (data not shown).

We noted that as a result of the effects of AMPK knockdown on the expression of the fat-forming enzymes, intracellular fat accumulation, which had been suppressed by the anti-adipogenic activity of $\beta$-HIVS, was significantly recovered by AMPK siRNA compared to transfection with the control siRNA (Fig. 7A and B). AMPK knockdown also reduced the effect of $\beta$-HIVS on the expression level of the major adipogenic transcription factors, PPAR $\gamma$ and $\mathrm{C} / \mathrm{EBP} \alpha$, and an adipocyte marker gene, FABP4, transcribed by them. The mRNA and protein levels of PPAR $\gamma, \mathrm{C} / \mathrm{EBP} \alpha$ and FABP4, which had been suppressed by $\beta$-HIVS, were significantly increased by AMPK knockdown (Fig. 7C-E).

The results of the present study demonstrated that AMPK is involved in the anti-adipogenic mechanism of $\beta$-HIVS through the modulation of SREBP-1c phosphorylation, maturation and nuclear translocation as well as the transcription of downstream fat-forming enzymes and adipogenic transcription factors. A possible molecular mechanism for the anti-adipogenic activity of $\beta$-HIVS and the effects of AMPK knockdown are described in Fig. 8.

\section{Discussion}

A number of natural compounds have the potential to exert anti-obesity effects through the inhibition of adipogenesis (26). As previously noted, $\beta$-HIVS is one of the natural shikonin compounds contained in a therapeutic plant, Lithospermum erythrorhizon (14). In the present study, modulations of AMPK and SREBP-1c were analyzed in relation to the anti-adipogenic mechanisms of $\beta$-HIVS, resulting in novel findings. Firstly, we noted that $\beta$-HIVS exerted the greatest anti-adipogenic effect of the four shikonin compounds. Secondly, $\beta$-HIVS effectively activated AMPK by increasing its phosphorylation at a much lower concentration, $<1 / 100,000$, compared with AICAR, a well-known AMPK activator. Thirdly, $\beta$-HIVS increased the phosphorylation of precursor SREBP-1c through the activation of AMPK, which prevented the cleavage of precursor SREBP-1c into mature SREBP-1c and its nuclear translocation. Accordingly, the expression of genes transcribed by mature SREBP-1c, which included fat-forming enzymes and an adipogenic transcription factor, were decreased, resulting in reduced intracellular fat accumulation. Finally, knockdown of AMPK attenuated the anti-adipogenic activity of $\beta$-HIVS, including the expression of fat-forming enzymes and an adipogenic transcription factor as well as intracellular fat accumulation, through the modulation of SREBP-1c.

AMPK is a serine/threonine kinase expressed in various tissues such as the skeletal muscle, liver and adipose tissue (27), and it regulates metabolic processes by upregulating catabolism and downregulating anabolism of lipid and carbohydrate (6-8). SREBPs are a family of transcription factors that regulate lipid homeostasis by controlling the expression of enzymes required for the synthesis of endogenous cholesterol, fatty acids, triacylglycerols and phospholipids. Accordingly, SREBPs have been identified as a novel therapeutic target for metabolic diseases such as insulin resistance, type 2 diabetes, fatty liver and atherosclerosis (28). The three SREBP isoforms, SREBP1a, SREBP-1c and SREBP2, play different roles in lipid metabolism. SREBP-1c is involved in fatty acid synthesis and lipogenesis, whereas SREBP2 and SREBP1a are mainly involved in cholesterol synthesis. SREBPs are synthesized as inactive precursors, and upon activation, the precursors undergo proteolytic cleavage to release the mature SREBPs into the nucleus (29). A previous study demonstrated AMPK-SREBP-1c modulation by revealing that activated AMPK directly phosphorylates precursor SREBP-1c at the Ser372 residue, which suppresses the proteolytic cleavage of precursor SREBP-1c into mature SREBP-1c and inhibits its nuclear translocation (11). 
Many phytochemicals, including resveratrol, epigallocatechin gallate, berberine, quercetin, rutaecarpine analogues and ursolic acid, have previously been reported to inhibit adipogenesis by activating AMPK but the molecular mechanisms downstream of AMPK have not been fully elucidated (30-32). It is noteworthy that until the present study, AMPK-SREBP-1c modulation has never been suggested as the anti-adipogenic mechanism of any compound which inhibits adipogenesis, to the best of our knowledge. In the present study, the detailed molecular mechanism for the anti-adipogenic activity of $\beta$-HIVS, which was found to be an efficient AMPK activator, was elucidated. $\beta$-HIVS activated AMPK to increase the phosphorylation of precursor SREBP-1c, which inhibited the formation of mature SREBP-1c, necessary for the transcription of fat-forming enzymes as well as a major transcription factor of adipogenesis.

\section{Acknowledgements}

The present study was supported by the Biomedical Science, Department of Medicine Research Scholarship Grants, of Chung-Ang University in 2014.

\section{References}

1. Grundy SM: Obesity, metabolic syndrome, and cardiovascular disease. J Clin Endocrinol Metab 89: 2595-2600, 2004.

2. Farmer SR: Transcriptional control of adipocyte formation. Cell Metab 4: 263-273, 2006.

3. Rosen ED and MacDougald OA: Adipocyte differentiation from the inside out. Nat Rev Mol Cell Biol 7: 885-896, 2006.

4. Rosen ED, Walkey CJ, Puigserver P and Spiegelman BM: Transcriptional regulation of adipogenesis. Genes Dev 14: 1293-1307, 2000.

5. Hardie DG, Ross FA and Hawley SA: AMPK: a nutrient and energy sensor that maintains energy homeostasis. Nat Rev Mol Cell Biol 13: 251-262, 2012.

6. Makinde AO, Gamble J and Lopaschuk GD: Upregulation of 5'-AMP-activated protein kinase is responsible for the increase in myocardial fatty acid oxidation rates following birth in the newborn rabbit. Circ Res 80: 482-489, 1997.

7. Ojuka EO, Jones TE, Nolte LA, Chen M, Wamhoff BR, Sturek M and Holloszy JO: Regulation of GLUT4 biogenesis in muscle: evidence for involvement of AMPK and $\mathrm{Ca}^{2+}$. Am J Physiol Endocrinol Metab 282: E1008-E1013, 2002.

8. Fazakerley DJ, Holman GD, Marley A, James DE, Stöckli J and Coster ACF: Kinetic evidence for unique regulation of GLUT4 trafficking by insulin and AMP-activated protein kinase activators in L6 myotubes. J Biol Chem 285: 1653-1660, 2010.

9. Mihaylova MM and Shaw RJ: The AMPK signalling pathway coordinates cell growth, autophagy and metabolism. Nat Cell Biol 13: 1016-1023, 2011.

10. Steinberg GR and Kemp BE: AMPK in health and disease. Physiol Rev 89: 1025-1078, 2009.

11. Li Y, Xu S, Mihaylova MM, Zheng B, Hou X, Jiang B, Park O, Luo Z, Lefai E, Shyy JY, et al: AMPK phosphorylates and inhibits SREBP activity to attenuate hepatic steatosis and atherosclerosis in diet-induced insulin-resistant mice. Cell Metab 13: 376-388, 2011.

12. Kim JB, Wright HM, Wright $M$ and Spiegelman BM: ADD1/ SREBP1 activates PPARgamma through the production of endogenous ligand. Proc Natl Acad Sci USA 95: 4333-4337, 1998.

13. Chen X, Yang L, Oppenheim JJ and Howard MZ: Cellular pharmacology studies of shikonin derivatives. Phytother Res 16: 199-209, 2002.
14. Ito $\mathrm{Y}$, Onobori $\mathrm{K}$, Yamazaki $\mathrm{T}$ and Kawamura Y: Tigloylshikonin, a new minor Shikonin derivative, from the roots and the commercial root extract of Lithospermum erythrorhizon. Chem Pharm Bull (Tokyo) 59: 117-119, 2011.

15. Li J, Zhang D, Ward KM, Prendergast GC and Ayene IS: Hydroxyethyl disulfide as an efficient metabolic assay for cell viability in vitro. Toxicol In Vitro 26: 603-612, 2012.

16. Gustafson B and Smith U: Cytokines promote Wnt signaling and inflammation and impair the normal differentiation and lipid accumulation in 3T3-L1 preadipocytes. J Biol Chem 281: 9507-9516, 2006.

17. Livak KJ and Schmittgen TD: Analysis of relative gene expression data using real-time quantitative PCR and the 2(-Delta Delta C(T)) method. Methods 25: 402-408, 2001.

18. Paton CM and Ntambi JM: Biochemical and physiological function of stearoyl-CoA desaturase. Am J Physiol Endocrinol Metab 297: E28-E37, 2009.

19. Shimomura I, Bashmakov Y, Ikemoto S, Horton JD, Brown MS and Goldstein JL: Insulin selectively increases SREBP-1c mRNA in the livers of rats with streptozotocin-induced diabetes. Proc Natl Acad Sci USA 96: 13656-13661, 1999.

20. Magaña MM, Lin SS, Dooley KA and Osborne TF: Sterol regulation of acetyl coenzyme A carboxylase promoter requires two interdependent binding sites for sterol regulatory element binding proteins. J Lipid Res 38: 1630-1638, 1997.

21. Fajas L, Schoonjans K, Gelman L, Kim JB, Najib J, Martin G, Fruchart JC, Briggs M, Spiegelman BM and Auwerx J: Regulation of peroxisome proliferator-activated receptor gamma expression by adipocyte differentiation and determination factor $1 /$ sterol regulatory element binding protein 1: implications for adipocyte differentiation and metabolism. Mol Cell Biol 19: 5495-5503, 1999.

22. Bijland S, Mancini SJ and Salt IP: Role of AMP-activated protein kinase in adipose tissue metabolism and inflammation. Clin Sci (Lond) 124: 491-507, 2013.

23. Lee H, Kang R, Bae S and Yoon Y: AICAR, an activator of AMPK, inhibits adipogenesis via the WNT/ $\beta$-catenin pathway in 3T3-L1 adipocytes. Int J Mol Med 28: 65-71, 2011.

24. Zhang C, Shin DJ and Osborne TF: A simple promoter containing two Sp1 sites controls the expression of sterol-regulatoryelement-binding protein 1a (SREBP-1a). Biochem J 386: 161-168, 2005.

25. Amemiya-Kudo M, Shimano H, Yoshikawa T, Yahagi N, Hasty AH, Okazaki H, Tamura Y, Shionoiri F, Iizuka Y, Ohashi K, et al: Promoter analysis of the mouse sterol regulatory element-binding protein-1c gene. J Biol Chem 275: 31078-31085, 2000.

26. Rayalam S, Della-Fera MA and Baile CA: Phytochemicals and regulation of the adipocyte life cycle. J Nutr Biochem 19: 717-726, 2008

27. Shirwany NA and Zou MH: AMPK in cardiovascular health and disease. Acta Pharmacol Sin 31: 1075-1084, 2010.

28. Xiao X and Song BL: SREBP: a novel therapeutic target. Acta Biochim Biophys Sin (Shanghai) 45: 2-10, 2013.

29. Eberlé D, Hegarty B, Bossard P, Ferré P and Foufelle F: SREBP transcription factors: master regulators of lipid homeostasis. Biochimie 86: 839-848, 2004.

30. Hwang JT, Kwon DY and Yoon SH: AMP-activated protein kinase: A potential target for the diseases prevention by natural occurring polyphenols. N Biotechnol 26: 17-22, 2009.

31. Chen YC, Zeng XY, He Y, Liu H, Wang B, Zhou H, Chen JW, Liu PQ, Gu LQ, Ye JM and Huang ZS: Rutaecarpine analogues reduce lipid accumulation in adipocytes via inhibiting adipogenesis/lipogenesis with AMPK activation and UPR suppression. ACS Chem Biol 8: 2301-2311, 2013.

32. He Y, Li Y, Zhao T, Wang Y and Sun C: Ursolic acid inhibits adipogenesis in 3T3-L1 adipocytes through LKB1/AMPK pathway. PLoS One 8: e70135, 2013. 